\title{
Constraints to Dark Matter from Inert Higgs Doublet Model
}

\author{
Marco Aurelio Díaz, Benjamin Koch, and Sebastián Urrutia-Quiroga \\ Instituto de Física, Pontificia Universidad Católica de Chile, Avenida Vicuña Mackenna 4860, Santiago, Chile \\ Correspondence should be addressed to Sebastián Urrutia-Quiroga; sgurruti@uc.cl
}

Received 19 November 2015; Revised 25 January 2016; Accepted 28 January 2016

Academic Editor: Michal Kreps

Copyright (C) 2016 Marco Aurelio Díaz et al. This is an open access article distributed under the Creative Commons Attribution License, which permits unrestricted use, distribution, and reproduction in any medium, provided the original work is properly cited. The publication of this article was funded by SCOAP ${ }^{3}$.

\begin{abstract}
We study the Inert Higgs Doublet Model and its inert scalar Higgs $H$ as the only source for dark matter. It is found that three mass regions of the inert scalar Higgs can give the correct dark matter relic density. The low mass region (between 3 and $50 \mathrm{GeV}$ ) is ruled out. New direct dark matter detection experiments will probe the intermediate (between 60 and $100 \mathrm{GeV}$ ) and high (heavier than $550 \mathrm{GeV}$ ) mass regions. Collider experiments are advised to search for $D^{ \pm} \rightarrow H W^{ \pm}$decay in the two jets plus missing energy channel.
\end{abstract}

\section{Introduction}

Astrophysical observations provide strong evidence for the existence of dark matter (DM) [1] and its abundance in the current phase of the Universe [2]. According to the newest results from Planck collaboration, there is $74 \%$ approximately of matter which is not directly visible but is observed due to its gravitational effects on visible matter. Additional evidence for the existence of DM comes from study of the rotation curves of spiral galaxies [3], the analysis of the bullet cluster [4], and the study of baryon acoustic oscillations [5]. One of the most common hypotheses used to explain these phenomena is to postulate the existence of weakly interacting massive particles (WIMPs) [6].

In order to explain DM, we study a simple extension of the Standard Model (SM), called the Inert Higgs Doublet Model (IHDM). This model, which was originally proposed for studies on electroweak (EW) symmetry breaking [7], introduces an additional doublet and a discrete symmetry. These two characteristics of the model modify the SM phenomenology, but there are some regions of the parameter space which predict only small deviations from the SM. Nevertheless, one of the most attractive characteristics of the IHDM is the presence of a stable neutral particle which can be a DM candidate.

In this work the IHDM is revisited, considering various restrictions, focusing our analysis on the zones of the parameter space which reproduce the correct DM relic density according to the newest measurements $[8,9]$. In this context, three not connected mass regimes for the lightest inert particle are found. These regimes are also analyzed using LHC observables like branching ratios to invisible particles and a specific SM-like Higgs boson decay mode. Additionally, we study some inert decays modes. Finally, we use a direct detection approach to rule out one of the mass regimes. It is further shown that this regime can also be ruled out by constraints from collider physics [10].

This paper is organized as follows. After a short introduction in Section 1 the model is introduced in Section 2 by formulating the associated potential and constraints of the model, and by exploring the parameter space and its characteristics in Section 3. In Section 4 the behavior of the model is presented from a collider physics perspective, studying the modifications of the SM and its implications for the IHDM. In Section 5 the results of this study are complemented by an analysis from the dark matter perspective. Finally, in Section 6 we remark on the most important conclusions of our work.

\section{The Inert Higgs Doublet Model}

Consider an extension of Standard Model (SM), which contains two Higgs doublets $\Phi_{S, D}$ and a discrete $\mathbb{Z}_{2}$ symmetry [7] $\left(\Phi_{S} \stackrel{\mathbb{Z}_{2}}{\longrightarrow} \Phi_{S}\right.$ and $\left.\Phi_{D} \stackrel{\mathbb{Z}_{2}}{\longrightarrow}-\Phi_{D}\right)$. All fields of the SM are 
invariants under the discrete symmetry, and $\Phi_{S}$ is completely analogous to the SM Higgs doublet.

The most general renormalizable $S U(2) \times U(1)$ invariant Higgs potential that also preserves the discrete symmetry is

$$
\begin{aligned}
V= & \mu_{1}^{2} \mathbf{A}+\mu_{2}^{2} \mathbf{B}+\lambda_{1} \mathbf{A}^{2}+\lambda_{2} \mathbf{B}^{2}+\lambda_{3} \mathbf{A} \mathbf{B}+\lambda_{4} \mathbf{C}^{\dagger} \mathbf{C} \\
& +\frac{\lambda_{5}}{2}\left(\mathbf{C}^{2}+\mathbf{C}^{\dagger 2}\right),
\end{aligned}
$$

where $\mathbf{A}, \mathbf{B}, \mathbf{C}$ are given by

$$
\begin{aligned}
& \mathbf{A}=\Phi_{S}^{\dagger} \Phi_{S}, \\
& \mathbf{B}=\Phi_{D}^{\dagger} \Phi_{D}, \\
& \mathbf{C}=\Phi_{S}^{\dagger} \Phi_{D} .
\end{aligned}
$$

The parameters $\mu_{i}^{2}(i=1,2)$ and $\lambda_{j}(j=1, \ldots, 4)$ are intrinsically real, and $\lambda_{5}$ will be assumed to be real [11]. After Spontaneous Symmetry Breaking, the vacuum expectation values of the Higgs doublets are

$$
\begin{aligned}
& \left\langle\Phi_{S}\right\rangle=\frac{1}{\sqrt{2}}\left(\begin{array}{l}
0 \\
v
\end{array}\right), \\
& \left\langle\Phi_{D}\right\rangle=\left(\begin{array}{l}
0 \\
0
\end{array}\right),
\end{aligned}
$$

where $\left\langle\Phi_{D}\right\rangle$ is forced by the discrete symmetry and $v=$ $246 \mathrm{GeV}$. Expanding the fields around those vacua, we define

$$
\begin{aligned}
& \Phi_{S}=\left(\begin{array}{c}
G^{+} \\
\frac{\left(v+h+i G^{0}\right)}{\sqrt{2}}
\end{array}\right), \\
& \Phi_{D}=\left(\begin{array}{c}
D^{+} \\
\frac{(H+i A)}{\sqrt{2}}
\end{array}\right) \text {, }
\end{aligned}
$$

where $G^{0}$ and $G^{ \pm}$are the neutral and charged Goldstone bosons and $h$ is the SM-like Higgs boson. The fields in the second doublet belong to the so-called dark or inert sector. They are the scalar $H$ and pseudoscalar $A$, both neutral, and the charged scalar $D^{ \pm}$. As in the SM, the parameter $\mu_{1}^{2}$ is related to $\lambda_{1}$ by the tree-level tadpole condition $\mu_{1}^{2}=-\lambda_{1} v^{2}$. The masses of physical states $[12,13]$ are

$$
\begin{aligned}
& m_{h}^{2}=2 \lambda_{1} v^{2}, \\
& m_{D}^{2}=\mu_{2}^{2}+\frac{\lambda_{3}}{2} v^{2}, \\
& m_{H}^{2}=m_{D}^{2}+\left(\frac{\lambda_{4}+\lambda_{5}}{2}\right) v^{2}=\mu_{2}^{2}+\frac{\lambda_{345}}{2} v^{2}, \\
& m_{A}^{2}=m_{D}^{2}+\left(\frac{\lambda_{4}-\lambda_{5}}{2}\right) v^{2},
\end{aligned}
$$

with

$$
\lambda_{345}=\lambda_{3}+\lambda_{4}+\lambda_{5} .
$$

As independent and free parameters we take the masses $m_{H}$, $m_{A}$, and $m_{D}$ at tree-level and the couplings $\lambda_{2}$ and $\lambda_{345}$. The SM-like Higgs boson mass is fixed now thanks to the measurement $m_{h}=125 \mathrm{GeV}[14,15]$.

The constraints we initially impose include vacuum stability at tree-level, where constraints on the $\lambda_{i}$ couplings and $\mu_{i}$ mass terms appear $[16-18]$; perturbation $\left(\left|\lambda_{i}\right|<8 \pi\right)[19,20]$ and unitarity [21], where we impose that the scalar potential is unitary and that several scattering processes between scalar and gauge bosons are bounded; electroweak precision tests through the $S, T$, and $U$ parameters [22] applied to the IHDM $[21,23]$, with $3 \sigma$ values given by

$$
\begin{gathered}
\left.S\right|_{U=0}=0.06 \pm 0.09 \\
\wedge \\
\left.T\right|_{U=0}=0.10 \pm 0.07,
\end{gathered}
$$

with a correlation coefficient of +0.91 [24]; and collider constraints [16, 25-27], where we satisfy lower bonds on the Higgs boson masses.

The DM particle must be neutral. In our analysis we assume it is the $H$ boson; thus $m_{H}<m_{A}$ and $m_{H}<m_{D}$, which due to (5) translates to

$$
\begin{gathered}
\lambda_{4}+\lambda_{5}<0 \\
\wedge \\
\lambda_{5}<0 .
\end{gathered}
$$

We do not consider $A$ as the DM candidate because it is analogous to consider $H$ as the DM candidate defining $\lambda_{345}^{-}=$ $\lambda_{3}+\lambda_{4}-\lambda_{5}$ instead of $\lambda_{345}$.

\section{IHDM Parameter Space}

We randomly scan the parameter space of the IHDM, taking into account all the constraints mentioned in the previous section. Additionally, we compute some astrophysical properties of the model using the micrOMEGAs software [28]. We consider masses satisfying $1 \mathrm{GeV}<m_{i}<1 \mathrm{TeV}$, where $i=H, A, D$. In addition, we consider cosmological measurements: the DM relic density $\Omega_{\mathrm{DM}} h^{2}$ is a property related to its abundance in the current phase of the Universe. This quantity is well measured by WMAP [29] and Planck [30] experiments. Following [31] to combine both measurements we obtain

$$
\Omega_{\mathrm{DM}} h^{2}=0.1181 \pm 0.0012 .
$$

In Figure 1 the coupling $\lambda_{345}$ is shown as a function of the Higgs boson mass $m_{H}$ varying $\left(\mu_{i}, \lambda_{i}, m_{A}, m_{D}\right.$, and $\left.m_{H}\right)$. We work with the hypothesis that the light inert Higgs boson $H$ is providing the complete DM density $\Omega_{\mathrm{DM}} h^{2}$ given in (9). The color code is as follows: red points (dark gray) produce 


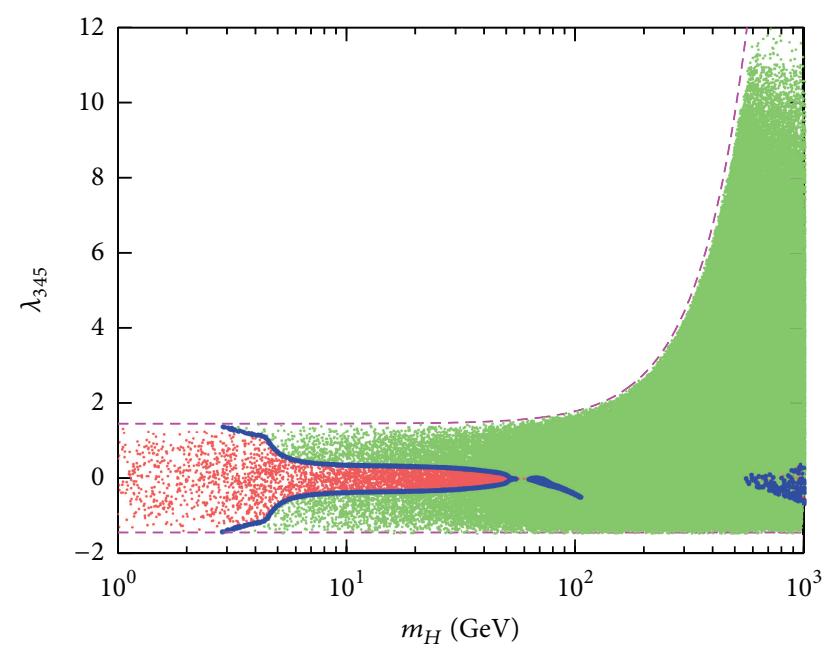

FIgURE 1: Random scan of IHDM parameter space. Coupling $\lambda_{345}$ as a function of the DM candidate mass $m_{H}$. The upper dotted line is the bound generated by the inert vacuum condition and the lower dotted line is generated by the vacuum stability condition.

a relic density above the $3 \sigma$ limit given in (9); blue points (black) produce a relic density within the $3 \sigma$ region; green points (light gray) produce a relic density below the $3 \sigma$ limit. Regarding the points that satisfy the relic density we see three clear regions [12, 32], one for low $m_{H}\left(3<m_{H}<50 \mathrm{GeV}\right)$, another one for medium $m_{H}\left(60<m_{H}<100 \mathrm{GeV}\right)$, and finally one for high values of $m_{H}\left(m_{H}>550 \mathrm{GeV}\right)$. The explanation for the gap is related to annihilation processes and it will be given later. At $m_{H}<3 \mathrm{GeV}$ the IHDM can no longer be compatible with vacuum existence and stability $[33,34]$.

In Figure 2 we have for the same scan and color code the mass of the heavy pseudoscalar $m_{A}$ (a) and the mass of the charged Higgs $m_{D}$ (b) as a function of the mass of the DM candidate $m_{H}$. Due to dedicated pre-LHC collider searches, a bound that captures most of the features is $m_{A}>100 \mathrm{GeV}$ and $m_{D}>70 \mathrm{GeV}$. This is so with the exception of a small strip for $m_{A}<100 \mathrm{GeV}$ seen in Figure 2(a), where due to the proximity of the $H$ and $A$ masses the search loses sensitivity. In this figure the gap in values of $m_{H}$ when the relic density is imposed is apparent. Notice that the density of solutions is larger when the masses for $H, A$, and $D$ are close to each other and that this feature is more pronounced when the masses of these particles are near the $\mathrm{TeV}$ scale (due to the logarithmic scale). Finally we notice that $\Omega_{\mathrm{DM}} h^{2}$ is more sensitive to the parameters $\lambda_{345}$ and $m_{H}$ (Figure 1) than the masses of the other inert particles (Figure 2).

\section{Collider Physics}

As we mentioned before, the Higgs boson discovered at CERN in 2012 is the SM-like Higgs boson $h$ of our model from the non-Inert Higgs Doublet field $\Phi_{s}$. This particle $h$ couples to the charged Higgs pair $\left(D^{ \pm}\right)$, which contributes to the diphoton decay width (in this minimal scenario, the IHDM cannot account for the reported excess of diphoton events by ATLAS [35] and CMS [36] collaborations in their Run-II $13 \mathrm{TeV}$ analyses, because the $\mathbb{Z}_{2}$ symmetry prevents the extra Higgs bosons of the model from decaying into just two photons) $\Gamma(h \rightarrow \gamma \gamma)$ [37]. For the same reason $D^{ \pm}$also contribute to $\Gamma(h \rightarrow Z \gamma)$.

It is convenient to work with the parameter $[21,33,38]$

$$
R_{\gamma \gamma}=\frac{B(h \longrightarrow \gamma \gamma)^{\mathrm{IHDM}}}{B(h \longrightarrow \gamma \gamma)^{\mathrm{SM}}} .
$$

The value we use for the $\mathrm{SM}$ is $\Gamma\left(h_{\mathrm{SM}} \rightarrow \gamma \gamma\right)=4.1 \mathrm{MeV}$ [39]. ATLAS [40] and CMS [41] collaborations have studied this decay mode, and if we combined both results [31] we obtain $R_{\gamma \gamma}^{\exp }=1.14 \pm 0.18$

In Figure 3 we have the parameter $R_{\gamma \gamma}$ as a function of the DM candidate mass $m_{H}$ (a) and as a function of the coupling $\lambda_{345}$ (b). The points in parameter space that produce a correct relic density can be divided into three groups. In the case of very light masses for the DM candidate $(3<$ $m_{H}<50 \mathrm{GeV}$ approximately) the decay mode $h \rightarrow \mathrm{HH}$ is open, and $R_{\gamma \gamma}$ is close to zero ruling those masses out [42]. In the intermediate mass case, approximately between 60 and $100 \mathrm{GeV}$, there is a region with acceptable solutions characterized by $R_{\gamma \gamma} \approx 1$. This region is characterized by increasingly heavier values for $A$ and $D^{ \pm}$. In the large mass region $\left(m_{H}>550 \mathrm{GeV}\right.$ approximately), the charged Higgs $D^{ \pm}$gives a negligible contribution to the decay $h \rightarrow \gamma \gamma$ such that $R_{\gamma \gamma}$ is close to unity. Interestingly, Figure 3(b) shows that perturbative values $\left(\left|\lambda_{345}\right|<1\right)$ are preferred.

In addition, if the inert particles are light enough, there are two other two-body decays which are

$$
\begin{aligned}
& \Gamma(h \longrightarrow H H)=\frac{v^{2} \lambda_{345}^{2}}{32 \pi m_{h}} \sqrt{1-\frac{4 m_{H}{ }^{2}}{m_{h}{ }^{2}}}, \\
& \Gamma(h \longrightarrow A A) \\
& \quad=\frac{\left(m_{A}{ }^{2}-m_{H}{ }^{2}+\lambda_{345} v^{2} / 2\right)^{2}}{8 \pi v^{2} m_{h}} \sqrt{1-\frac{4 m_{A}{ }^{2}}{m_{h}{ }^{2}}} .
\end{aligned}
$$

There is no phase space for a two-body decay $h \rightarrow D^{ \pm} D^{\mp}$. It is possible to define the parameter $R_{Z \gamma}$, in analogy to $R_{\gamma \gamma}$ defined in (10). It is interesting that even though the decay $h \rightarrow Z \gamma$ is not well measured, it still can give additional insight to the model $[43,44]$. As Figure 4 shows, there appears a very narrow correlation between $R_{\gamma \gamma}$ and $R_{Z \gamma}$, which is a common feature for $R_{\gamma \gamma}$ versus $R_{Z \gamma}$ plots $[17,18,45]$. The bisector branch only contains points that satisfy $m_{H}<m_{h} / 2$ (inert invisible decay channel open). Points of parameter space which satisfy the relic density and have low DM candidate mass are ruled out, because they produce a very small value for $R_{\gamma \gamma}$. By analyzing the characteristics of those data points one finds that the larger branch includes only points with $m_{H}>m_{h} / 2$, and the ones that also satisfy relic density are close to $R_{\gamma \gamma}=R_{Z \gamma}=1$, as was mentioned before. The two branches seen in the $R_{\gamma \gamma}$ versus $R_{Z \gamma}$ relation also appear in the nonnormalized $B(h \rightarrow \gamma \gamma)$ versus $B(h \rightarrow Z \gamma)$ relation (not shown). But it is reduced only to the long (green) 


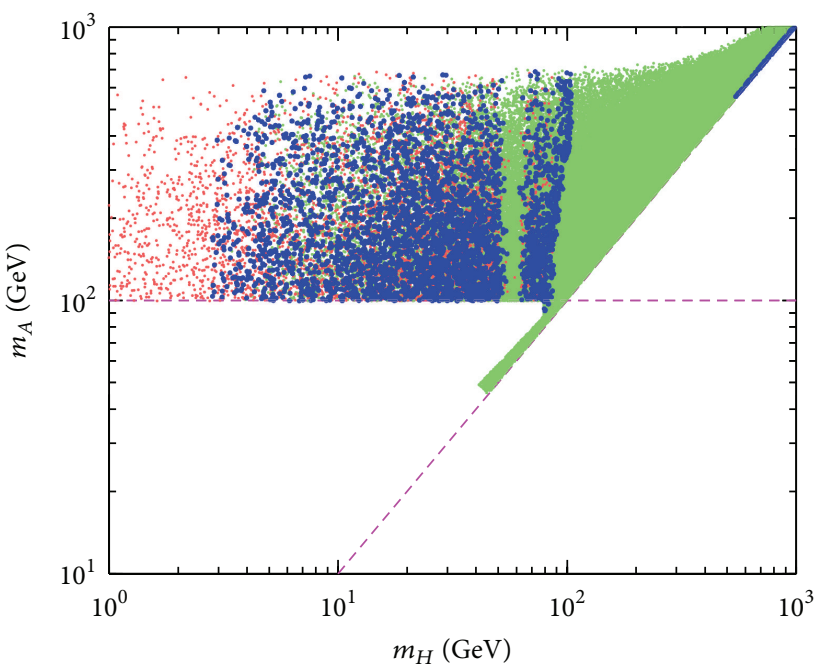

(a)

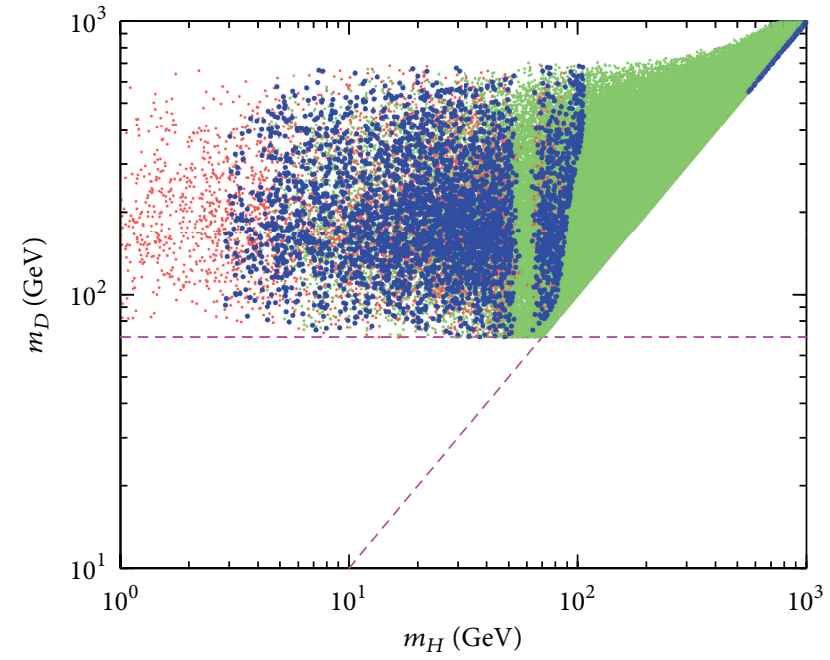

(b)

FiguRE 2: Inert Higgs masses $m_{A}$ (a) and $m_{D}$ (b) as a function of the DM candidate mass $m_{H}$, for the same random scan of IHDM parameter space. The horizontal dotted line is due to LEP constraints and the diagonal dotted line is due to the $m_{H}$-DM condition.

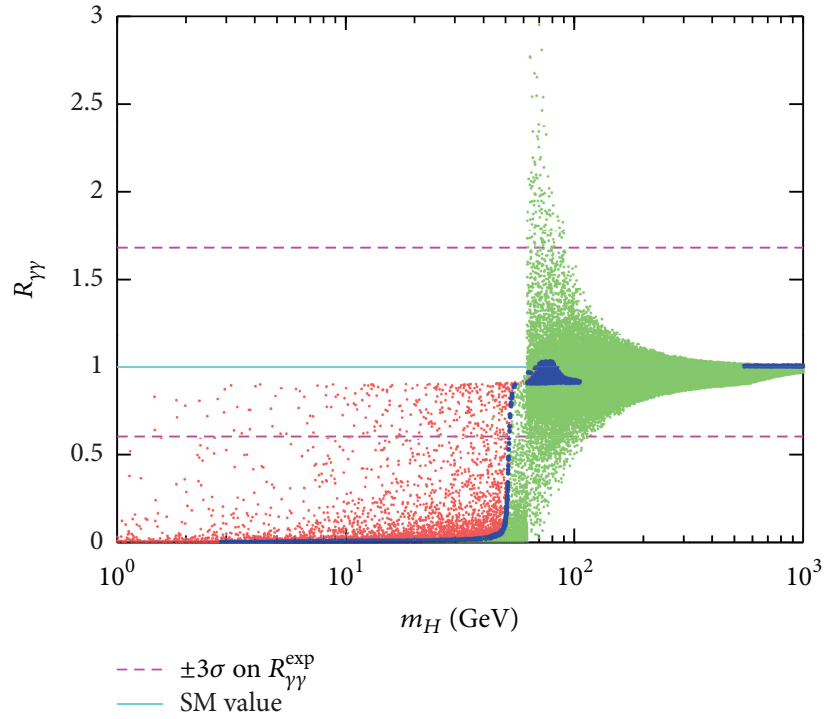

(a)

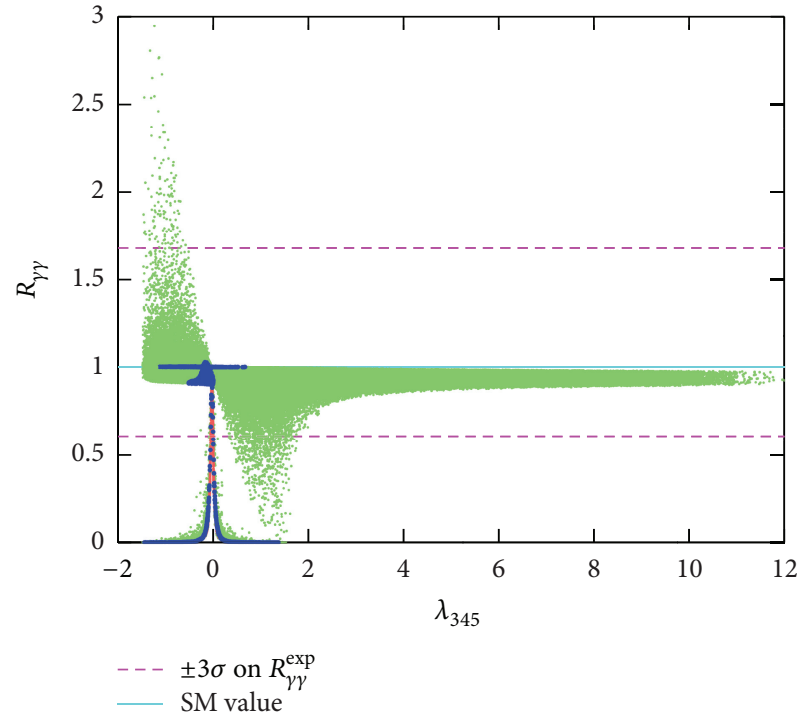

(b)

FIGURE 3: $R_{\gamma \gamma}$ parameter as a function of the DM candidate mass $m_{H}$ (a) and the $\lambda_{345}$ coupling (b), for the same random scan of IHDM parameter space.

branch in the $\Gamma(h \rightarrow \gamma \gamma)$ versus $\Gamma(h \rightarrow Z \gamma)$ relation. Further one sees that when the $h \rightarrow H H$ decay channel is closed, the $D^{ \pm}$loop transforms into the long branch, the otherwise SM dot (the intersection point between the two branches). If the $h \rightarrow H H$ decay channel is open, the second branch appears because the $h \rightarrow H H$ channel tends to dominate [46].

We are also interested in the invisible decay of the SM-like Higgs boson. If the DM candidate mass satisfies $m_{H}<m_{h} / 2$, the two-body decay channel $h \rightarrow H H$ is open, which is invisible for the LHC detectors and shows only as missing momentum. There are measurements for the invisible decay of the SM-like Higgs from the LHC experiments. Taking a simple average of the upper bounds to the invisible decay rate from ATLAS [47] and CMS [48] gives $B(h \rightarrow$ inv $)<0.43$ for the SM-like Higgs boson. In Figure 5 we show the branching ratio for the invisible decay of the SM-like Higgs boson $B(h \rightarrow$ $H H)$, as a function of the mass of the DM candidate $m_{H}$ (a) and as a function of the parameter $R_{\gamma \gamma}$ (b). In (a) we also have a horizontal line that shows the upper bound for $B(h \rightarrow H H)$ mentioned above. The threshold $2 m_{H}=m_{h}$ appears clearly in (a). Most of the points with correct relic density satisfying $m_{H}<60 \mathrm{GeV}$ are ruled out because they produce a very large invisible branching ratio for $h$. On the contrary, most of the points with higher DM candidate mass 


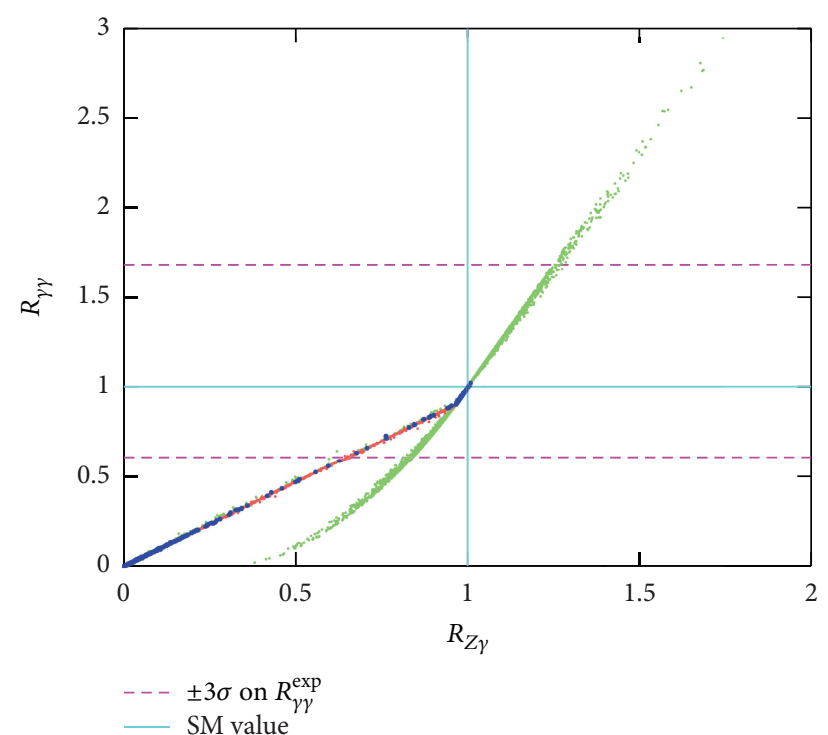

FIgURE 4: Relation between $R_{\gamma \gamma}$ and $R_{Z \gamma}$ for the same random scan of IHDM parameter space.

are fine because they produce an invisible branching ratio equal to zero. In (b), where we have as dashed lines the bounds from LHC experiments, we see a very strong relation between $B(h \rightarrow H H)$ and $R_{\gamma \gamma}$. The points that satisfy relic density with a low mass for the DM candidate are simultaneously excluded from $B(h \rightarrow H H)$ and from $R_{\gamma \gamma}$. The rest of the points satisfy the bounds.

To finalize this section we discuss the branching ratios for the two observable inert Higgs bosons $A$ and $D^{ \pm}$. In Figure 6 we have the branching ratios for the pseudoscalar Higgs boson (a) as a function of its mass and the branching ratios for the charged Higgs boson (b) as a function of its mass. In (a) we show the decays of the inert pseudoscalar Higgs $A$, which are $A \rightarrow H Z$ (solid line) and $A \rightarrow D^{ \pm} W^{\mp}$ (dashed line). As can be seen from the Feynman rules, the only unknown parameters the branching ratios depend on are the masses $m_{H}, m_{A}$, and $m_{D}$. Therefore, four scenarios are considered: (i) $\left(m_{H}, m_{D}\right)=(60,70)$, (ii) $(450,500)$, (iii) $(50,600)$, and (iv) $(500,800) \mathrm{GeV}$. The gauge boson can be off shell, although we consider the inert Higgs bosons always on shell. The oscillation near the threshold is due to different increasing rates for the decay rates when the gauge boson is off shell. The branching ratio $B(A \rightarrow H Z)$ is always large (because $\left.m_{H}<m_{D}\right)$ while $B\left(A \rightarrow D^{ \pm} W^{\mp}\right)$ can be low near thresholds. There is a crossing point where $B(A \rightarrow H Z)=$ $B\left(A \rightarrow D^{ \pm} W^{\mp}\right)$. In (b) we show the decays of the charged Higgs $D^{ \pm}$. Analogous scenarios are considered, but replacing $m_{D}$ by $m_{A}$. In solid line we have the branching ratio for the decay $D^{ \pm} \rightarrow H W^{ \pm}$and in dash we have $D^{ \pm} \rightarrow A W^{ \pm}$. In the case of $D^{ \pm}$, there is no crossing point; thus $B\left(D^{ \pm} \rightarrow H W^{ \pm}\right)$ is always larger than $B\left(D^{ \pm} \rightarrow A W^{ \pm}\right)$. We remind the reader that the presence of a Higgs boson $H$ in a final state is seen as missing momentum at the LHC.

\section{Cosmology and Dark Matter}

The existence of dark matter seems to be well established now [49]. There are several candidates for DM; among them are the previously mentioned WIMPs. A good particle candidate for DM must be neutral and stable (or quasi-stable). The $\mathbb{Z}_{2}$ discrete symmetry in the model studied in this paper ensures that the lightest of the inert Higgs bosons is stable. Observation implies it is either $H$ or $A$ (or in a fine tuned scenario both). In this paper we study the former case. An important restriction this candidate must satisfy is that its mass density must agree with experimental observations. We calculate the relic density of our DM candidate using micrOMEGAs software [28]. To better understand the results on the relic density, we calculate also the thermal averaged annihilation cross section times the relative velocity $\langle\sigma v\rangle$, or annihilation cross section for short.

In Figure 7 we plot the $H$ relic density as a function of the DM candidate mass $m_{H}(\mathrm{a})$, and the annihilation cross section also as a function of the DM candidate mass $m_{H}(\mathrm{~b})$, calculated with [28]. For the relic density case, we also show, as an horizontal dashed line, the experimentally measured value for $\Omega_{\mathrm{DM}} h^{2}$ as given in (9). The scan shows a large distribution with differences that can reach more than 10 orders of magnitude. For this reason most of the points in the scan are ruled out if one demands that $H$ is actually the only WIMP responsible for the observed DM signatures. There are two mass gaps that divide the mass region in three: low mass ( $3<m_{H}<50 \mathrm{GeV}$ approximately), medium mass $\left(60<m_{H}<100 \mathrm{GeV}\right.$ approximately), and high mass (550< $m_{H}$ approximately). The origin of these mass gaps is better understood with the aid of the right frame. In the right frame of Figure 7 we show the annihilation cross section as a function of the DM candidate mass $m_{H}$, with vertical lines denoting different thresholds. The first gap is near the threshold $m_{H} \approx m_{h} / 2$ where the annihilation channel $\mathrm{HH} \rightarrow h$ becomes very efficient due to the fact that the SM-like Higgs $h$ is on-shell. The second gap starts at the thresholds $m_{H} \approx m_{W}$ and $m_{H} \approx m_{Z}$, where $H H \rightarrow$ $W W(Z Z)$ become available, and continues later with the threshold $m_{H} \approx m_{h}$ where the channel $\mathrm{HH} \rightarrow h h$ opens up. The annihilation channel $H H \rightarrow t \bar{t}$ also helps. All these new annihilation channels make the DM annihilation very efficient, and it is not possible to obtain a relic density according to observations. On the other hand, for larger $m_{H}$, it is possible to get a correct relic density if the difference between the three inert scalar masses is not so large and $\lambda_{345}$ remains small enough [50] (see Figure 1).

We finally study the direct detection prospects of our DM candidate. We do that through the tree-level spinindependent DM-nucleon interaction cross section [51], which applied to our case,

$$
\sigma_{\mathrm{DM}-N}^{\mathrm{SI}}=\frac{\lambda_{345}^{2}}{\left(4 \pi m_{h}^{4}\right)} \frac{m_{N}^{4} f_{N}^{2}}{\left(m_{H}+m_{N}\right)^{2}} .
$$

Here $m_{h}$ is the mass of the SM-like Higgs boson, $m_{H}$ is the mass of the DM candidate, $m_{N}$ is the nucleon mass, taken here to be $m_{N}=0.939 \mathrm{GeV}$ as the average of the proton 


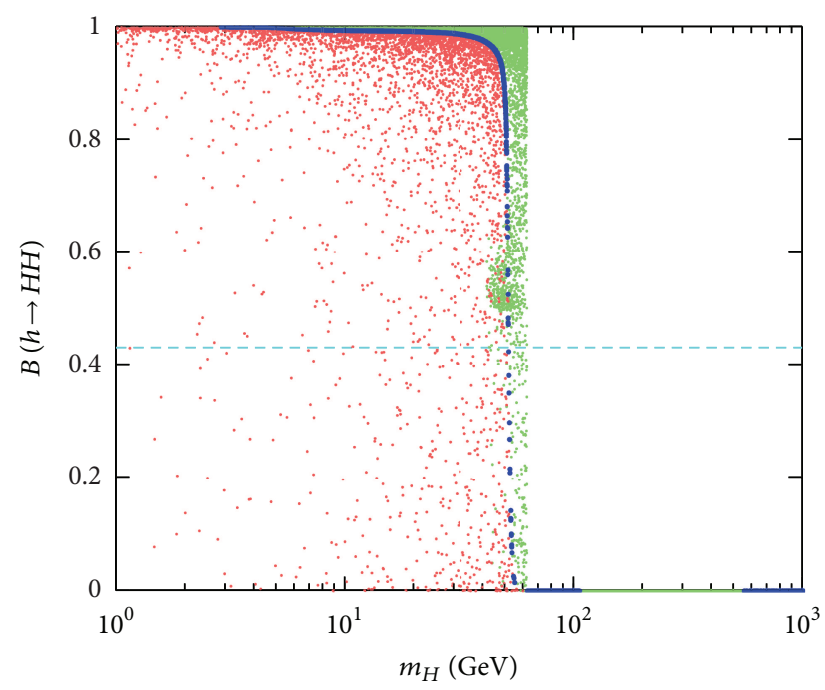

(a)

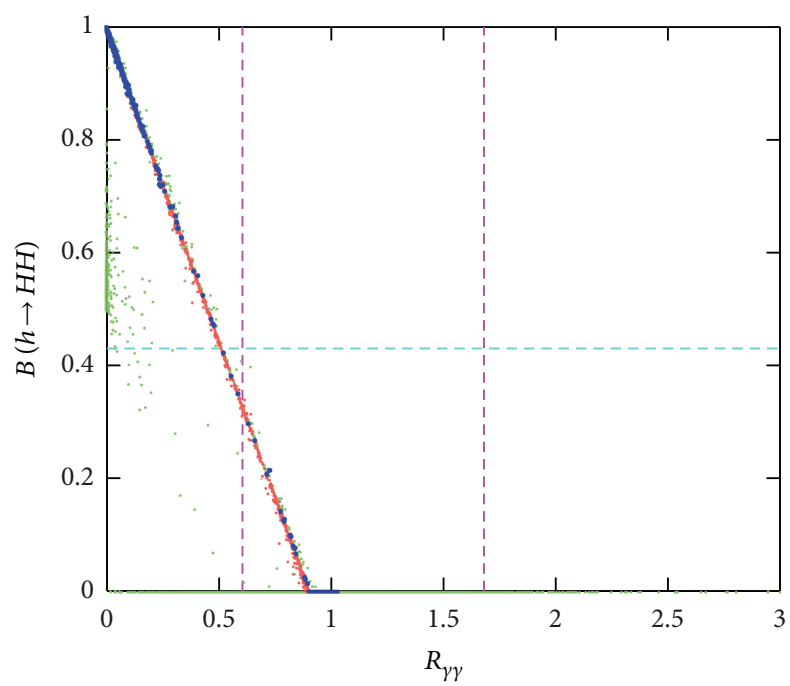

(b)

FIGURE 5: Branching ratio for the invisible decay of the SM-like Higgs boson as a function of the DM candidate mass $m_{H}$ (a) and as a function of the parameter $R_{\gamma y}$ (b), for the same random scan of IHDM parameter space. Please note that there is a number of points on the $m_{H}$ and $R_{\gamma \gamma}$ axes, with $B(h \rightarrow H H)=0$.

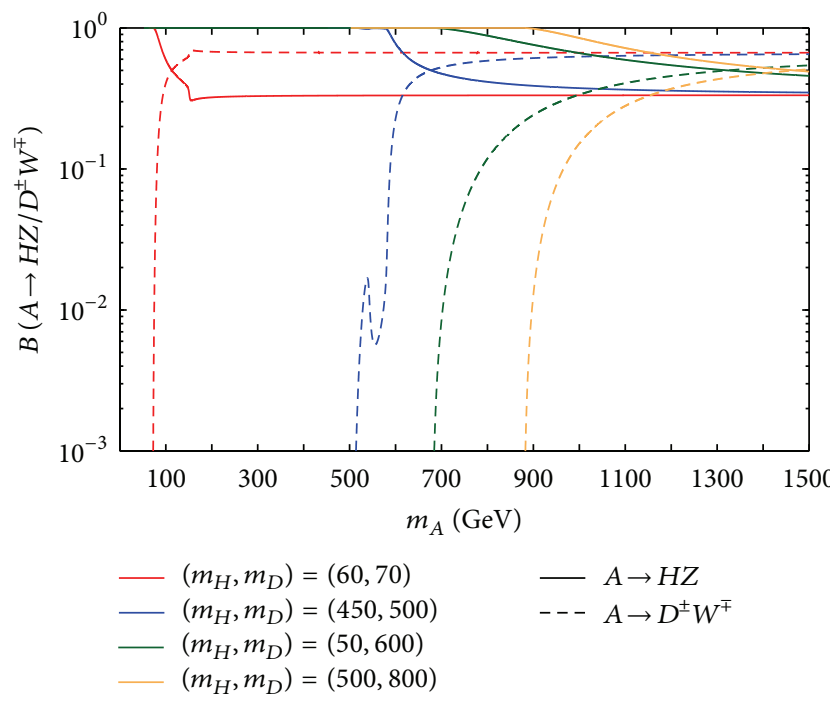

(a)

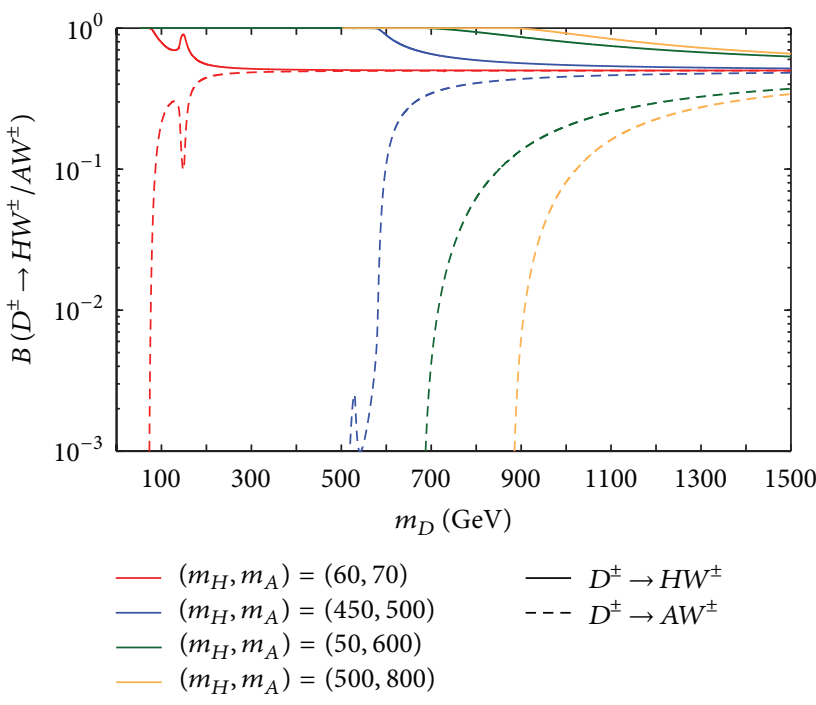

(b)

FIgURE 6: Branching ratios for the inert Higgs bosons $A(\mathrm{a})$ and $D^{ \pm}$(b) as a function of their corresponding mass.

and neutron masses, $\lambda_{345}$ is the combined coupling defined in (6), and $f_{N}$ is a form factor that depends on hadronic matrix elements $[52,53]$.

In Figure 8 we show the DM-nucleon cross section as a function of the DM candidate mass for a correct value of $\Omega_{\mathrm{DM}} h^{2}$. We consider three values for $f_{N}$ : a central value (0.326) from a lattice calculation [54], and extreme values (0.260 and 0.629) from the MILC collaboration [55]. Lower bounds for past experiments and prospects of measurements for future experiments are also shown [56-59]. Notice that the dispersion of points for high $m_{H}$ can be understood from analogous dispersion seen in Figure 1, and the same situation occurs with the line-like distribution for light $m_{H}$. We also show the coherent neutrino scattering upper limit [60]. This curve represents the threshold below which the detector sensitivity is such that not only can the possible DM scattering effects be observed, but also the indistinguishable scattering effects are associated with neutrinos. Thus, this indicates a region where the neutrino background becomes dominant and little information can be obtained on DM effects. Current direct detection of DM excludes all the low DM mass points, and most of the medium DM mass points. Allowed are a narrow region near $60 \mathrm{GeV}$ and all the high mass region. Note that the absence of points in the range of $\sim 100-550 \mathrm{GeV}$ in 


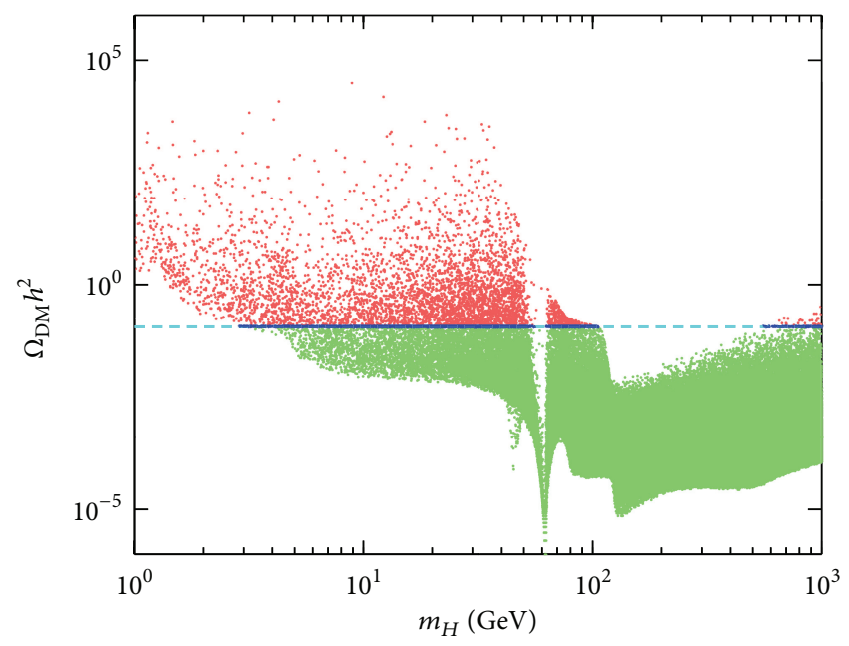

(a)

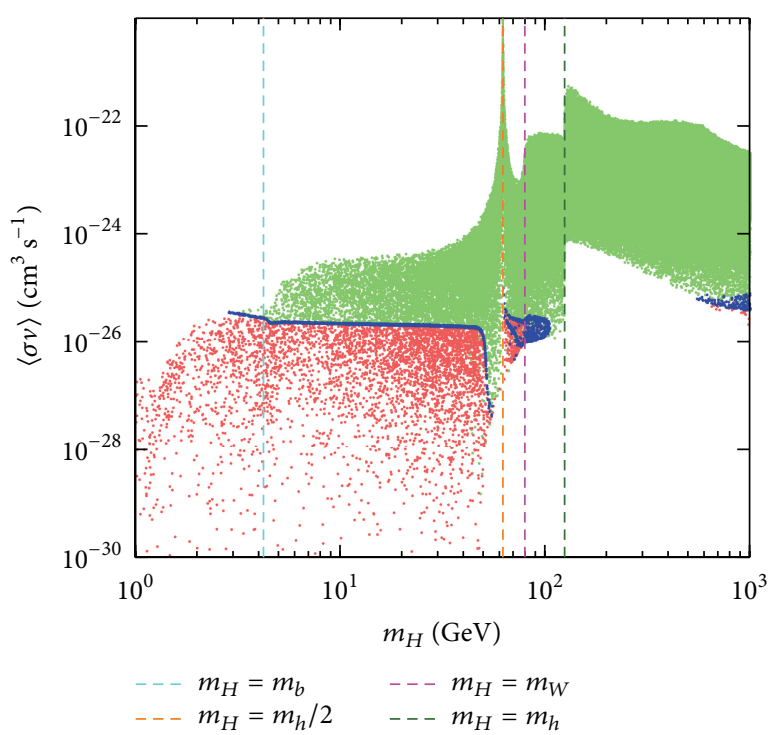

(b)

Figure 7: Relic density for $H$ as a function of its mass $m_{H}$ (a), and annihilation cross section for $H$ also as a function of its mass $m_{H}$ (b), for the same random scan of IHDM parameter space.

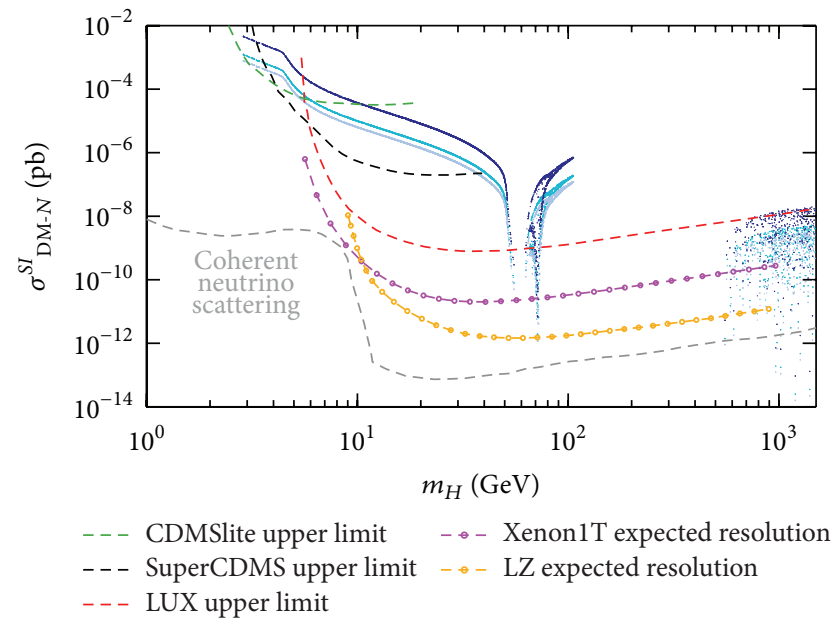

FIGURE 8: Spin-independent DM-nucleon cross section as a function of the DM candidate mass, for points of the IHDM with DM relic density consistent with observation. Three scenarios are considered with different values for the form factor $f_{N}$. Lower bounds for past experiments and prospects of measurements for future experiments are shown.

this plot is due to the fact that the plotted points are only those that give the right dark matter density ("blue points"). Future experiments will be able to test large parts of these two regions but will not be able to rule them out entirely if there is no signal.

\section{Conclusions}

In this paper the Inert Higgs Doublet Model is studied, with the inert Higgs boson $H$ as a DM candidate, using the latest results for DM relic density, annihilation cross section, and collider searches. As a summary we highlight the following:

(i) The branching ratios for the charged Higgs $D^{ \pm}$and for the pseudoscalar Higgs $A$ are studied and shown in Figure 6. The $\mathbb{Z}_{2}$ symmetry strongly reduces the number of different decay channels. Considering the Higgs boson on shell and allowing the gauge boson to be off shell (a different choice would produce different decay channels, but with smaller branching ratios), we find that $B\left(D^{ \pm} \rightarrow H W^{ \pm}\right)>B\left(D^{ \pm} \rightarrow A W^{ \pm}\right)$as opposed to the $A$ decays, where there is a crossing point not far from the threshold. For this reason, in collider searches we recommend to look for a signal for a $D^{ \pm}$: two jets (consistent with a $W$ ) and missing energy (from the DM candidate $H$ ).

(ii) Three distinct $H$ mass regions are found that produced the correct relic density (i.e., $H$ is the only source for DM) as can be seen in Figure 7. The low mass region (between 3 and $50 \mathrm{GeV}$ approximately) is already ruled out because it produces a very small value for $R_{\gamma \gamma}$ (Figure 3 ), because it produces a very high value for $B(h \rightarrow H H)$ (Figure 5 ) and because of direct DM searches (Figure 8). The intermediate mass region (between 60 and $100 \mathrm{GeV}$ approximately) and the high mass region (heavier than $550 \mathrm{GeV}$ approximately) are allowed.

(iii) In Figure 8 we study the DM candidate direct detection. The low mass region is also ruled out by present experiments. In addition, future experiments will probe intermediate and high mass regions. Nevertheless, in absence of signals, it will not be possible to rule out these two regions. Notice the proximity of 
the region where the coherent neutrino scattering is an irreducible background.

With this one sees that the Inert Higgs Doublet Model gives a still viable DM candidate, which will most likely be tested by direct DM detection experiments.

\section{Conflict of Interests}

The authors declare that there is no conflict of interests regarding the publication of this paper.

\section{Acknowledgments}

The authors are grateful for conversations with Drs. Germán Gómez, Nicolás Viaux, and Edson Carquín. Marco Aurelio Díaz was partly supported by Fondecyt 1141190. Benjamin Koch was partly supported by Fondecyt 1120360. Sebastián Urrutia-Quiroga was partly supported by postgraduate CONICYT grant. The work of all of the authors was also partly financed by CONICYT Grant ANILLO ACT-1102.

\section{References}

[1] D. Clowe, M. Bradač, A. H. Gonzalez et al., "A direct empirical proof of the existence of dark matter," The Astrophysical Journal, vol. 648, no. 2, pp. L109-L113, 2006.

[2] P. A. R. Ade, N. Aghanim, M. I. Alves et al., "Planck 2013 results. I. Overview of products and scientific results," Astronomy \& Astrophysics, vol. 571, article A1, 2014.

[3] V. C. Rubin, W. K. Ford Jr., and N. Thonnard, "Rotational properties of $21 \mathrm{SC}$ galaxies with a large range of luminosities and radii, from NGC $4605 / \mathrm{R}=4 \mathrm{kpc} /$ to UGC $2885 / \mathrm{R}=$ 122 kpcl," Astrophysical Journal, vol. 238, pp. 471-487, 1980.

[4] M. Markevitch, A. H. Gonzalez, D. Clowe et al., "Direct constraints on the dark matter self-interaction cross-section from the merging galaxy cluster 1E0657-56," The Astrophysical Journal, vol. 606, no. 2, pp. 819-824, 2004.

[5] W. J. Percival, S. Cole, D. J. Eisenstein et al., "Measuring the baryon acoustic oscillation scale using the sloan digital sky survey and $2 \mathrm{dF}$ galaxy redshift survey," Monthly Notices of the Royal Astronomical Society, vol. 381, no. 3, pp. 1053-1066, 2007.

[6] C. J. Copi, D. N. Schramm, and M. S. Turner, "Big-bang nucleosynthesis and the baryon density of the universe," Science, vol. 267, no. 5195, pp. 192-199, 1995.

[7] N. G. Deshpande and E. Ma, "Pattern of symmetry breaking with two Higgs doublets," Physical Review D, vol. 18, no. 7, pp. 2574-2576, 1978 .

[8] N. Blinov, J. Kozaczuk, D. E. Morrissey, and A. de la Puente, "Compressingthe inert doublet model," http://arxiv.org/ abs/1510.08069.

[9] N. Khan and S. Rakshit, "Constraints on inert dark matter from the metastability of the electroweak vacuum," Physical Review D, vol. 92, no. 5, Article ID 055006, 2015.

[10] G. Bélanger, B. Dumont, A. Goudelis, B. Herrmann, S. Kraml, and D. Sengupta, "Dilepton constraints in the inert doublet model from Run 1 of the LHC," Physical Review D, vol. 91, no. 11, Article ID 115011, 2015.

[11] I. F. Ginzburg, K. A. Kanishev, M. Krawczyk, and D. Sokolowska, "Evolution of Universe to the present inert phase," Physical Review D, vol. 82, Article ID 123533, 2010.
[12] L. Lopez Honorez and C. E. Yaguna, "The inert doublet model of dark matter revisited," Journal of High Energy Physics, vol. 2010, no. 9, article 046, 2010.

[13] A. Arhrib, Y. L. S. Tsai, Q. Yuan, and T. C. Yuan, "An updated analysis of Inert Higgs Doublet Model in light of the recent results from LUX, PLANCK, AMS-02 and LHC," Journal of Cosmology and Astroparticle Physics, vol. 2014, no. 6, article 30, 2014.

[14] G. Aad, T. Abajyan, B. Abbott et al., "Observation of a new particle in the search for the Standard Model Higgs boson with the ATLAS detector at the LHC," Physics Letters B, vol. 716, no. 1, pp. 1-29, 2012.

[15] S. Chatrchyan et al., "Observation of a new boson with mass near $125 \mathrm{GeV}$ in pp collisions at $\sqrt{S}=7$ and $8 \mathrm{TeV}$," Journal of High Energy Physics, vol. 2013, article 81, 2013.

[16] M. Gustafsson, "The inert doublet model and its phenomenology," in Proceedings of the Prospects for Charged Higgs Conference (PoS CHARGED '10), vol. 30, 2010, http://arxiv .org/abs/1106.1719.

[17] E. C. F. S. Fortes, A. C. B. Machado, J. Montaño, and V. Pleitez, "Prediction of $h \rightarrow \gamma Z$ from $h \rightarrow \gamma \gamma$ at the LHC for the $\mathrm{IMDS}_{3}$ model," Journal of Physics G: Nuclear and Particle Physics, vol. 42, no. 11, Article ID 115001, 2015.

[18] M. Krawczyk, D. Sokołowska, P. Swaczyna, and B. Świeżewska, "Higgs $\rightarrow \gamma \gamma, Z \gamma$ in the inert doublet model," Acta Physica Polonica B, vol. 44, no. 11, pp. 2163-2170, 2013.

[19] R. Barbieri, L. J. Hall, and V. S. Rychkov, "Improved naturalness with a heavy Higgs boson: an alternative road to CERN LHC physics," Physical Review D, vol. 74, no. 1, Article ID 015007, 2006.

[20] A. Djouadi, "The anatomy of electroweak symmetry breaking: tome I: the Higgs boson in the Standard Model," Physics Reports, vol. 457, no. 1-4, pp. 1-216, 2008.

[21] A. Arhrib, R. Benbrik, and N. Gaur, " $H \rightarrow \gamma \gamma$ in the inert Higgs doublet model," Physical Review D, vol. 85, no. 9, Article ID 095021, 2012.

[22] M. E. Peskin and T. Takeuchi, "Estimation of oblique electroweak corrections," Physical Review D, vol. 46, no. 1, pp. 381409, 1992.

[23] B. Swiezewska, "Yukawa independent constraints for twoHiggs-doublet models with a $125 \mathrm{GeV}$ Higgs boson," Physical Review D, vol. 88, no. 5, Article ID 055027, 2013, Erratum: Physical Review D, vol. 88, no. 11, Article ID 119903, 2013.

[24] M. Baak, J. Cúth, J. Haller et al., "The global electroweak fit at NNLO and prospects for the LHC and ILC," The European Physical Journal C, vol. 74, article 3046, pp. 1-14, 2014.

[25] E. Lundström, M. Gustafsson, and J. Edsjö, "Inert doublet model and LEP II limits," Physical Review D, vol. 79, no. 3, Article ID 035013, 2009.

[26] Q. H. Cao, E. Ma, and G. Rajasekaran, "Observing the dark scalar doublet and its impact on the standard-model Higgs boson at colliders," Physical Review D, vol. 76, no. 9, Article ID 095011, 2007.

[27] A. Pierce and J. Thaler, "Natural Dark Matter from an unnatural Higgs boson and new colored particles at the TeV scale," Journal of High Energy Physics, vol. 2007, no. 8, article 026, 2007.

[28] G. Belanger, F. Boudjema, and A. Pukhov, "micrOMEGAs: a code for the calculation of Dark Matter properties in generic models of particle interaction," http://arxiv.org/abs/1402.0787.

[29] C. L. Bennett, D. Larson, J. L. Weiland et al., "Nine-year Wilkinson Microwave Anisotropy Probe (WMAP) observations: final 
maps and results," The Astrophysical Journal Supplement Series, vol. 208, no. 2, article 20, 2013.

[30] P. A. R. Ade, N. Aghanim, M. Arnaud et al., "Planck 2015 results. XIII.Cosmological parameters," http://arxiv.org/abs/ 1502.01589 .

[31] G. Bohm and G. Zech, Introduction to Statistics and Data Analysis for Physicists, Deutsches Elektronen-Synchrotron, Hamburg, Germany, 2010.

[32] L. Lopez Honorez and C. E. Yaguna, "A new viable region of the inert doublet model," Journal of Cosmology and Astroparticle Physics, vol. 2011, no. 1, article 002, 2011.

[33] A. Goudelis, B. Herrmann, and O. Stål, "Dark matter in the inert doublet model after the discovery of a Higgs-like boson at the LHC," Journal of High Energy Physics, vol. 2013, article 106, 2013.

[34] M. Cirelli, N. Fornengo, and A. Strumia, "Minimal dark matter," Nuclear Physics B, vol. 753, no. 1-2, pp. 178-194, 2006.

[35] ATLAS Collaboration, "Search for resonances decaying to photon pairs in $3.2 \mathrm{fb}^{-1}$ of pp collisions at $\sqrt{s}=13 \mathrm{TeV}$ with the ATLAS detector," ATLAS-CONF-2015-081.

[36] CMS Collaboration, "Search for new physics in high mass diphoton events in proton-proton collisions at $\sqrt{s}=13 \mathrm{TeV}$," CMS-PAS-EXO-15-004, 2015.

[37] J. F. Gunion, H. E. Haber, G. L. Kane, and S. Dawson, "The Higgs hunter's guide," Frontiers of Physics, vol. 80, pp. 1-448, 2000.

[38] M. Krawczyk, D. Sokolowska, P. Swaczyna, and B. Swiezewska, "Constraining Inert Dark Matter by $R_{\gamma \gamma}$ and WMAP data," Journal of High Energy Physics, vol. 2013, article 55, 2013.

[39] A. Djouadi, J. Kalinowski, and M. Spira, "HDECAY: a program for Higgs boson decays in the Standard Model and its supersymmetric extension," Computer Physics Communications, vol. 108, no. 1, pp. 56-74, 1998.

[40] G. Aad, B. Abbott, J. Abdallah et al., "Measurement of Higgs boson production in the diphoton decay channel in $p p$ collisions at center-of-mass energies of 7 and $8 \mathrm{TeV}$ with the ATLAS detector," Physical Review D, vol. 90, no. 11, Article ID 112015, 2014.

[41] V. Khachatryan, A. M. Sirunyan, A. Tumasyan et al., "Precise determination of the mass of the Higgs boson and tests of compatibility of its couplings with the standard model predictions using proton collisions at 7 and $8 \mathrm{TeV}$," The European Physical Journal C, vol. 75, no. 5, article 212, 2015.

[42] A. Ilnicka, M. Krawczyk, and T. Robens, "The Inert Doublet Model in thelight of LHC and astrophysical data-an update," http://arxiv.org/abs/1508.01671.

[43] G. Aad, T. Abajyan, B. Abbott et al., "Search for Higgs boson decays to a photon and a $Z$ boson in $p p$ collisions at $\sqrt{S}=7$ and $8 \mathrm{TeV}$ with the ATLAS detector," Physics Letters B, vol. 732, pp. 8-27, 2014.

[44] S. Chatrchyan, V. Khachatryan, A. M. Sirunyan et al., "Search for a Higgs boson decaying into a $\mathrm{Z}$ and a photon in pp collisions at $\sqrt{s}=7$ and 8 TeV," Physics Letters B, vol. 726, no. 4-5, pp. 587-609, 2013.

[45] C. S. Chen, C. Q. Geng, D. Huang, and L. H. Tsai, "New scalar contributions to $h \rightarrow Z \gamma$," Physical Review D, vol. 87, no. 7, Article ID 075019, 2013.

[46] B. Świeżewska and M. Krawczyk, "Diphoton rate in the inert doublet model with a $125 \mathrm{GeV}$ Higgs boson," Physical Review D, vol. 88, no. 3, Article ID 035019, 2013.

[47] G. Aad, B. Abbott, J. Abdallah et al., "Search for invisible decays of a Higgs boson using vector-boson fusion in pp collisions at $\sqrt{S}=8 \mathrm{TeV}$ with the ATLAS detector," Journal of High Energy Physics, vol. 2016, article 172, 2016.
[48] S. Chatrchyan, V. Khachatryan, A. M. Sirunyan et al., "Search for invisible decays of Higgs bosons in the vector boson fusion and associated ZH production modes," The European Physical Journal C, vol. 74, article 2980, 2014.

[49] K. A. Olive, K. Agashe, C. Amsler et al., "Review of particle physics," Chinese Physics C, vol. 38, no. 9, Article ID 090001, 2014.

[50] T. Hambye, F.-S. Ling, L. Lopez Honorez, and J. Rocher, "Scalar multiplet dark matter," Journal of High Energy Physics, vol. 2009, no. 7, article 090, 2009, Erratum in: Journal of High Energy Physics, vol. 2010, no. 5, article 066, 2010.

[51] A. Djouadi, O. Lebedev, Y. Mambrini, and J. Quevillon, "Implications of LHC searches for Higgs-portal dark matter," Physics Letters B, vol. 709, no. 1-2, pp. 65-69, 2012.

[52] S. Kanemura, S. Matsumoto, T. Nabeshima, and N. Okada, "Can WIMP dark matter overcome the nightmare scenario?" Physical Review D, vol. 82, no. 5, Article ID 055026, 2010.

[53] J. M. Alarcón, L. S. Geng, J. Martin Camalich, and J. A. Oller, "The strangeness content of the nucleon from effective field theory and phenomenology," Physics Letters B, vol. 730, pp. 342346, 2014.

[54] R. D. Young and A. W. Thomas, "Octet baryon masses and sigma terms from an SU(3) chiral extrapolation," Physical Review D, vol. 81, Article ID 014503, 2010.

[55] D. Toussaint and W. Freeman, "Strange quark condensate in the nucleon in $2+1$ flavor QCD," Physical Review Letters, vol. 103, no. 12, Article ID 122002, 2009.

[56] D. S. Akerib, H. M. Araújo, X. Bai et al., "First results from the LUX dark matter experiment at the sanford underground research facility," Physical Review Letters, vol. 112, no. 9, Article ID 091303, 2014.

[57] R. Agnese, A. J. Anderson, M. Asai et al., "Search for lowmass weakly interacting massive particles using voltage-assisted calorimetric ionization detection in the superCDMS experiment," Physical Review Letters, vol. 112, no. 4, Article ID 041302, 2014.

[58] R. Agnese, A. J. Anderson, M. Asai et al., "Search for lowmass weakly interacting massive particles with SuperCDMS," Physical Review Letters, vol. 112, no. 24, Article ID 241302, 2014.

[59] R. Gaitskell, M. De Jesus, and L. Roszkowski, Dark Matter Tools Workgroup, University of California, Berkeley, Berkeley, Calif, USA, http://dmtools.berkeley.edu.

[60] J. Billard, E. Figueroa-Feliciano, and L. Strigari, "Implication of neutrino backgrounds on the reach of next generation dark matter direct detection experiments," Physical Review D, vol. 89, no. 2, Article ID 023524, 2014. 

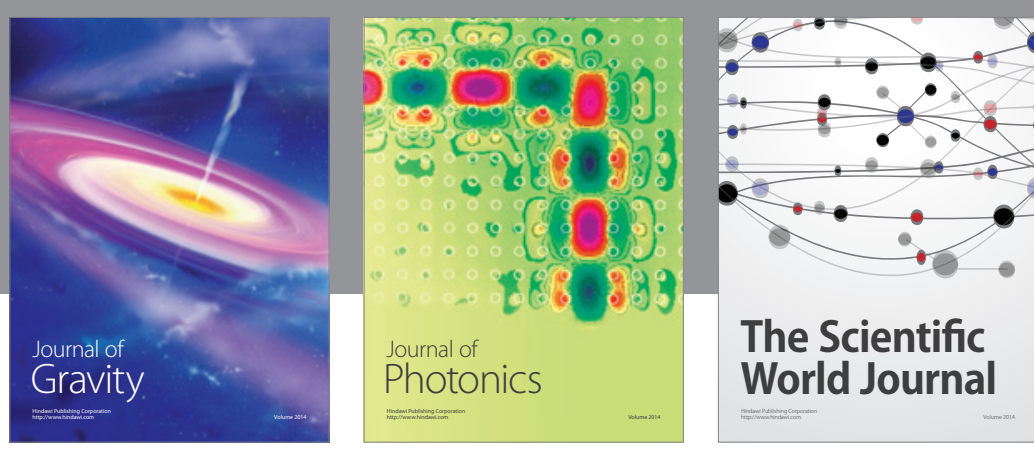

The Scientific World Journal
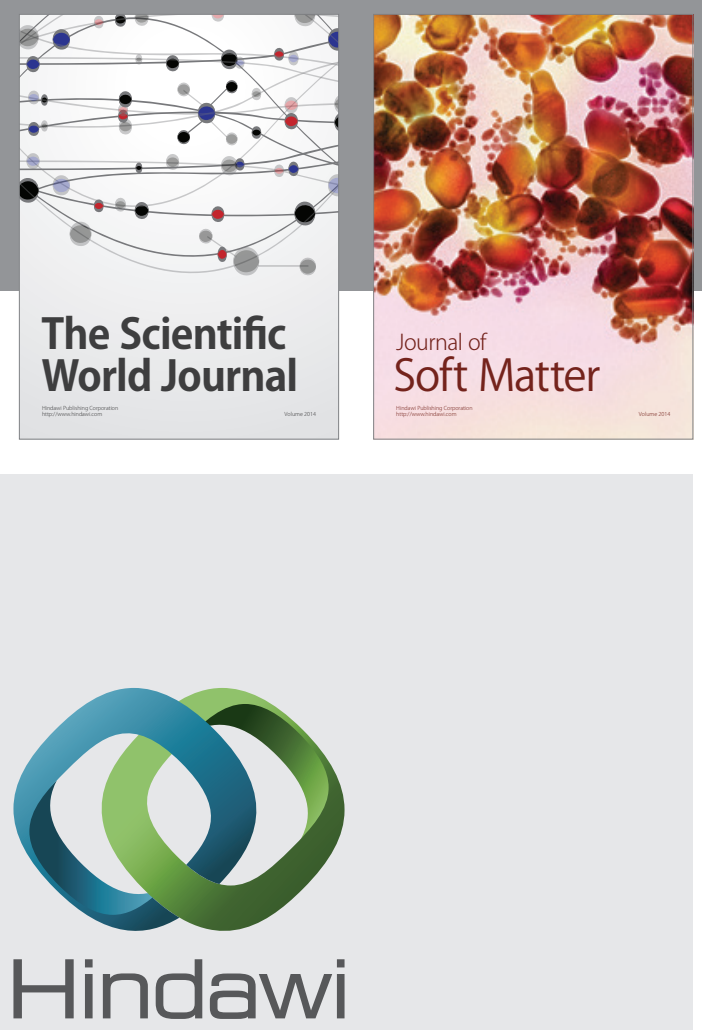

Submit your manuscripts at

http://www.hindawi.com

nternational Journal of

Statistical Mechanics
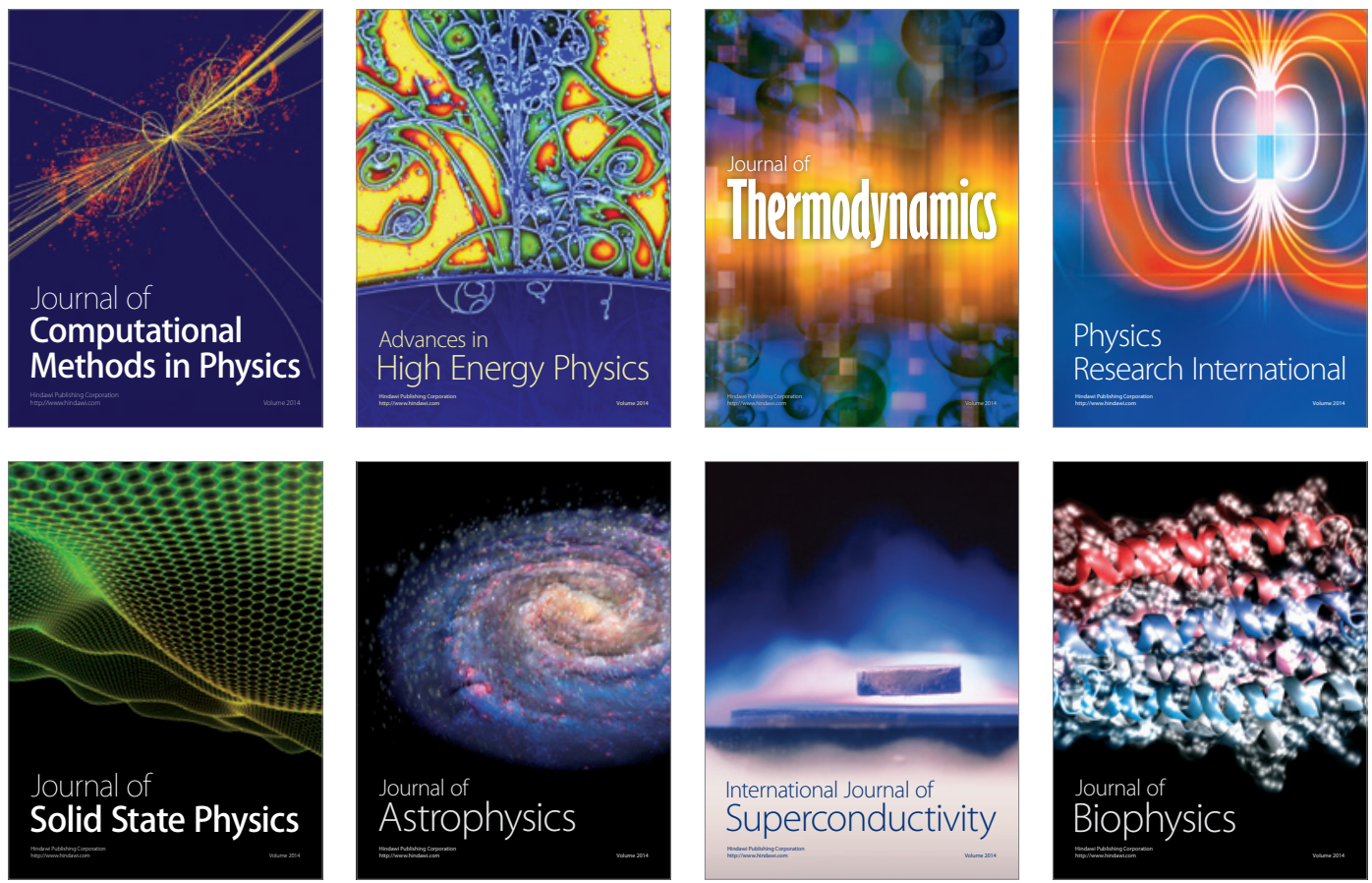
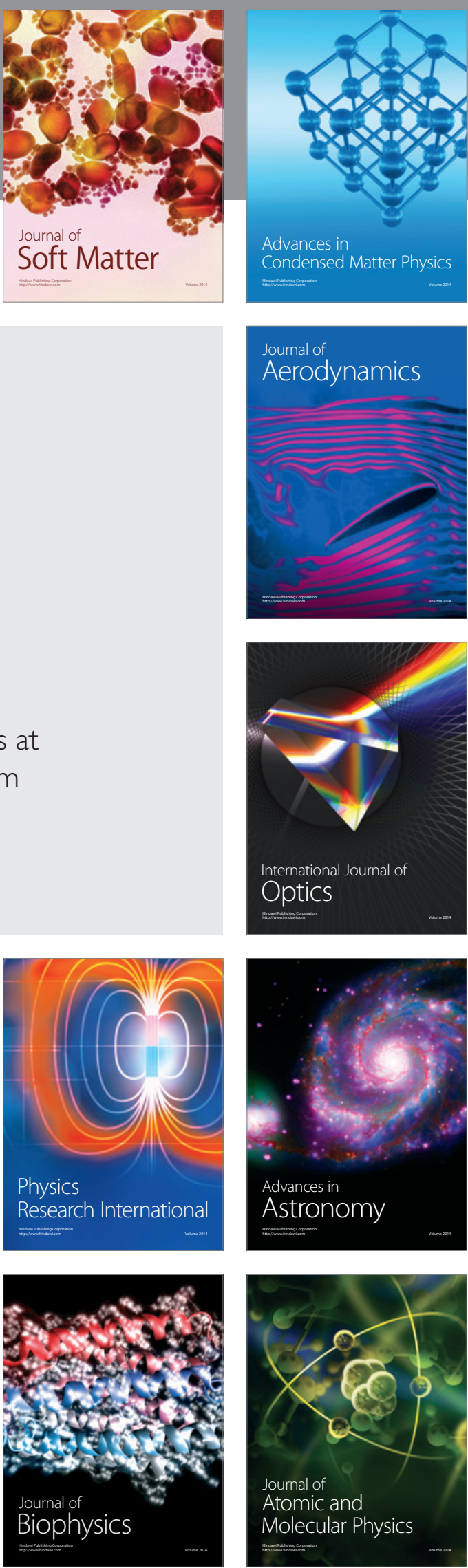